\title{
Evaluation of a continuous community-based ITN distribution pilot in Lainya County, South Sudan 2012-2013
}

Albert Kilian ${ }^{1,2^{*}}$, Lisa Woods Schnurr ${ }^{3}$, Tafadzwa Matova ${ }^{3}$, Richmond Ato Selby ${ }^{1,4}$, Kojo Lokko ${ }^{4}$, Sean Blaufuss ${ }^{4}$, Miatta Zenabu Gbanya ${ }^{3}$, Ruth Allan ${ }^{3}$, Hannah Koenker ${ }^{4}$, Martin Swaka ${ }^{5}$, George Greer ${ }^{6}$, Megan Fotheringham ${ }^{5}$, Lilia Gerberg ${ }^{5}$ and Matthew Lynch ${ }^{3}$

\begin{abstract}
Background: Continuous distribution of insecticide-treated nets (ITNs) has now been accepted as one way of sustaining ITN universal coverage. Community-based channels offer an interesting means of delivering ITNs to households to sustain universal ITN coverage. The objective of this study was to provide proof of concept for this channel.

Methods: A 9-month, community-based, distribution pilot was implemented beginning 1 year after a mass campaign in Lainya County, South Sudan from 2012 to 2013. Following social mobilization, community members could request an ITN from a net coupon holder. Eligibility criteria included having lost an ITN, giving birth outside of the health facility, or not having enough ITNs for all household members. After verification, households could exchange the coupon for an ITN at a distribution point. The evaluation was a pre/post design using representative household surveys with two-stage cluster sampling and a sample size of 600 households per survey.

Results: At endline, $78 \%$ of respondents were aware of the scheme and $89 \%$ of those also received an ITN through community-based distribution. Population access to ITNs nearly doubled, from 38\% at baseline to $66 \%$ after the pilot. Household ownership of any ITN and enough ITNs (1 for 2 people) also increased significantly, from 66 to $82 \%$ and 19 to $46 \%$, respectively. Community-based distribution was the only source of ITNs for $53.4 \%$ of households. The proportion of the population using an ITN last night increased from $22.7 \%$ at baseline to $53.9 \%$ at endline. A logistic regression model indicates that although behaviour change communication was positively associated with an increase in ITN use, access to enough nets was the greatest determinant of use.

Conclusions: ITN access and use improved significantly in the study area during the pilot, coming close to universal coverage targets. This pilot serves as proof of concept for the community-based distribution methodology implemented as a mechanism to sustain ITN universal coverage. Longer periods of implementation should be evaluated to determine whether community-based distribution can successfully maintain ITN coverage beyond the short term, and reach all wealth quintiles equitably.
\end{abstract}

Keywords: Insecticide-treated net, Malaria, South Sudan, Community-based, Insecticide-treated net distribution

\section{Background}

With the shift from conventionally treated mosquito nets to long-lasting insecticidal nets (LLIN), which avoid the need for retreatment, insecticide-treated nets (ITNs)

\footnotetext{
*Correspondence: albert@trophealth.com

${ }^{2}$ Tropical Health LLP, Montagut, Spain

Full list of author information is available at the end of the article
}

have become the most important tool in malaria control in Africa south of the Sahara. Initially distribution strategies were focused on local, often targeted distributions by non-government organizations [1] and some social marketing programmes [2], resulting in only slow improvements in coverage [3]. This changed around 2004 when ITN distributions started to be linked to mass immunization campaigns such as measles [4] or polio [5]. 
Following the 2007 launch of the new WHO universal coverage strategy for ITNs [6], campaigns changed from being mostly targeted to young children and pregnant women to mass campaigns for the entire population at risk. From 2008 to 2016 more than one billion ITNs have been delivered to African countries and distributed using a variety of approaches to mass campaign organization which in general were successful in rapidly and equitably scaling up ITN coverage if properly managed and implemented [7]. A 2015 study analysing factors contributing to declines in malaria morbidity between 2010 and 2015 found that $68 \%$ of the $40 \%$ reduction in malaria incidence could be attributed to ITNs [8].

However, it is clear that access to ITNs starts to decrease immediately following a mass campaign, due to births, migration and net loss or damage. Coverage can reduce to levels as low as $40 \%$ before the next campaign, potentially resulting in reduction or loss of the community effect [9]. In addition, it is not possible to take existing nets into account during mass campaigns [10], meaning that older but still serviceable nets and those distributed through ante-natal care and immunization services are ignored [6]. These attributes of mass campaigns can result in an oversupply of households with nets [11] and thereby reduce the cost-effectiveness of an ITN distribution strategy that includes repeat mass campaigns.

Comprehensive continuous distribution strategies have been suggested as a mechanism to sustain universal coverage once scale-up is achieved with mass campaigns, and include school and community-based channels in addition to routine distribution via health services and support to commercial market development [12]. However, data are limited about the ability of these strategies to increase or maintain ITN ownership and access. In South Sudan, the malaria control programme and partners distributed approximately 6 million LLINs through mass campaigns between 2008 and 2010 but faced significant challenges due to shortage of staff and weakness of health system infrastructure. Schooling rates in this complex operating environment of South Sudan are low, suggesting that a school-based distribution channel for ITN would not reach enough households to maintain universal coverage. Therefore, the government expressed interest in community-based ITN distribution. The purpose of this pilot study was to test the hypothesis that a demanddriven, community-based ITN distribution scheme would be able to at least sustain the level of coverage achieved through a preceding mass distribution campaign of ITN.

\section{Methods}

\section{Study site}

Lainya County was selected jointly by the South Sudan malaria control programme and implementation partners based on criteria of accessibility, safety and available minimal infrastructure. Lainya County is situated in southern South Sudan in Central Equatorial State with an estimated population in 2011 of 246,000 divided into five payams (3rd level administrative unit) and 15 bomas (4th level administrative unit). It had at the time one functional hospital, four health centres and 16 health units served by a total of 29 health staff. A mass distribution of ITNs took place in the County in 2010, but the exact number of nets distributed in Lainya County is not available.

\section{Continuous distribution of LLINs Design}

The community-based ITN distribution was based on a 'push-pull' system, where ITNs were delivered to a hub near the community (push), here primarily health facilities. Distribution then was driven by the expressed demand from households (pull). Households were informed about the distribution scheme through a social mobilization and communication campaign and encouraged to request new or additional nets if they felt they needed them. Three eligibility criteria were established:

1. An existing ITN that was too damaged and had to be replaced;

2. Less than one ITN for every two people was available in the household;

3. A birth occurred outside a health facility, and/or no ITN was received during ANC services for that pregnancy.

In order to receive an ITN through the communitybased distribution scheme, the household first made a claim to a community agent, called the net coupon holder. The net coupon holder then visited the household and authenticated the claim based on the eligibility criteria. A net coupon holder for every 700 households was trained to support the scheme. After the claim was verified, a coupon was issued to the household. Each coupon was valid for only one ITN but households could be issued multiple coupons. Household members were then able to redeem the coupon for an ITN at a distribution point. Initially, nine health facilities served as distribution points, but five additional remote distribution sites were added to improve distribution point access based on the request of communities to have distribution points closer to them. Since no adequate storage facilities were available at these sites, safe storage boxes were provided. There was then one distribution point for approximately every 2500 households. All distribution sites were supplied by the Lainya County store. Figure 1 illustrates how information, ITNs and coupons flow in the scheme. 


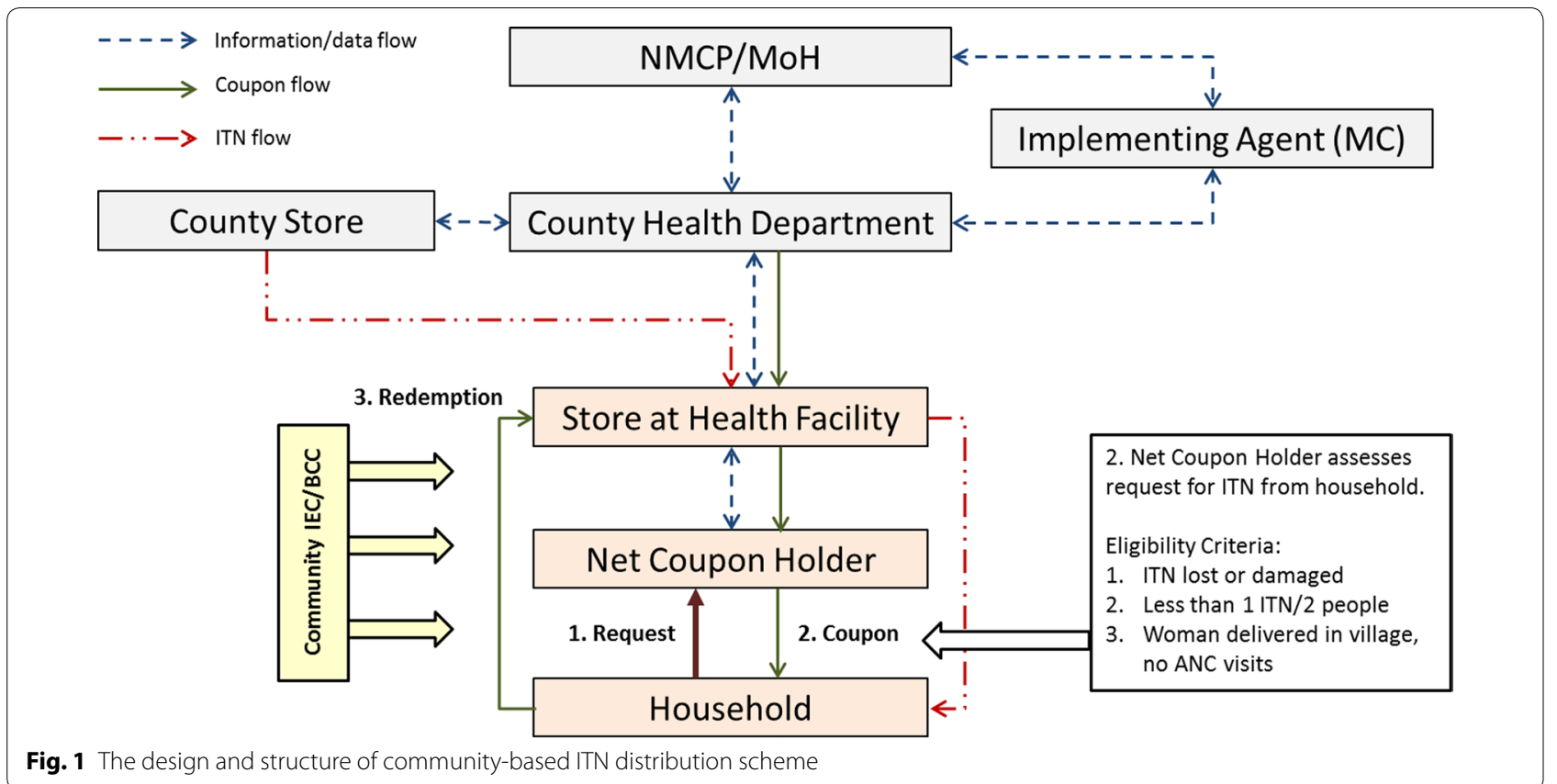

\section{Implementation}

The implementation of the scheme was led by the Lainya County health department and the South Sudan malaria control programme. Malaria Consortium provided implementation support and coordination as well as handling funds and initial transport logistics for ITNs. All ITNs were LLINs (PermaNet 2.0 or NetProtect) and were donated by UNICEF and Population Services International (PSI) via Global Fund or procured directly through Malaria Consortium. In addition to the 50 net coupon holders, there were 11 supervisors and 19 social mobilizers identified by the Lainya health department. Each store had a storekeeper and eight stores also had an assistant storekeeper to ensure access at all times. All stores were supervised by the central storekeeper, and monthly supervision and coordination meetings were held between the Lainya County Health Department and the implementing partner.

Activities started in December 2011 with training, social mobilization, and a SBCC (social and behavioural change communication) campaign that included community meetings with involvement of political and faithbased leaders. Coupon issuing started in May 2012 and lasted for 9 months until January 2013, while coupon redemption lasted until mid-March 2013.

\section{Evaluation}

The principle design was a before-after comparison based on household surveys with complementary routine and cost data. There was no external comparison or control group, but a counterfactual was obtained from the endline survey calculating outcomes without the ITNs distributed through the scheme.

\section{Routine data}

Two pieces of data were collected on a monthly basis: the number of ITNs issued at distribution points and the number of net coupons issued by net coupon holders. Net coupon holders and health facilities disseminated information upwards to the County health department, and finally to Malaria Consortium and the Ministry of Health. The data were entered into Excel data sheets. Information on the coupons also included the reason for issuing the coupon based on the three eligibility criteria.

\section{Household surveys}

Representative household surveys were carried out in April 2012 (baseline) and April 2013 (endline) each coinciding with the beginning of the rains, which last from April to October in this area. A two-stage cluster design was used where 30 clusters were selected based on enumeration areas from the 2008 Census and applying probability proportionate to size sampling (population). Within selected clusters, 20 households were sampled by simple random sampling from lists of eligible households compiled by the survey team (Table 1). The sample size of 600 households per survey was calculated to give a precision of $\pm 6 \%$-points for household indicators if the estimate was around $50 \%$ assuming a non-response rate of $5 \%$ and design effect of 2.0, alpha error of 0.05 and beta-error of 
Table 1 Background characteristics of sampled households (HH)

\begin{tabular}{|c|c|c|c|c|}
\hline \multirow[t]{2}{*}{ Category/variable } & \multicolumn{2}{|l|}{ Baseline } & \multicolumn{2}{|l|}{ Endline } \\
\hline & Estimate & $95 \% \mathrm{Cl}$ & Estimate & $95 \% \mathrm{Cl}$ \\
\hline \multicolumn{5}{|l|}{ Demographics } \\
\hline $\begin{array}{l}\text { Average household } \\
\text { size (persons) }\end{array}$ & 5.8 & $5.5-6.1$ & 5.1 & $4.9-5.3$ \\
\hline $\mathrm{HH}$ headed by female & $15.3 \%$ & $12.8-18.3$ & $17.2 \%$ & $14.0-21.0$ \\
\hline $\begin{array}{l}\text { Mean age of head of } \\
\text { HH (years) }\end{array}$ & 41.5 & $40.5-42.5$ & 42.0 & $40.9-43.2$ \\
\hline Population $<5$ years & $20.2 \%$ & $17.6-22.9$ & $15.3 \%$ & $14.1-16.6$ \\
\hline \multicolumn{5}{|l|}{ House characteristics } \\
\hline Thatch or grass roof & $96.2 \%$ & $93.8-97.7$ & $95.5 \%$ & $92.8-97.4$ \\
\hline Mud walls & $98.5 \%$ & $96.0-99.4$ & $97.2 \%$ & $95.1-98.3$ \\
\hline $\begin{array}{l}\text { Firewood primary fuel } \\
\text { for cooking }\end{array}$ & $93.2 \%$ & $86.6-96.4$ & $96.3 \%$ & $92.2-98.3$ \\
\hline $\begin{array}{l}\text { Average persons/ } \\
\text { sleeping place }\end{array}$ & 2.0 & $1.9-2.1$ & 1.5 & $1.4-1.5$ \\
\hline \multicolumn{5}{|c|}{ Education of head of $\mathrm{HH}$} \\
\hline Males non-literate & $20.2 \%$ & $24.8-33.9$ & $30.6 \%$ & $25.2-36.5$ \\
\hline Females non-literate & $76.7 \%$ & $65.9-84.3$ & $58.2 \%$ & $47.9-67.7$ \\
\hline $\begin{array}{l}\text { Males secondary or } \\
\text { higher }\end{array}$ & $21.9 \%$ & $18.0-26.5$ & $19.2 \%$ & $15.6-23.5$ \\
\hline $\begin{array}{l}\text { Females secondary or } \\
\text { higher }\end{array}$ & $2.2 \%$ & $0.5-8.6$ & $14.3 \%$ & $8.2-23.7$ \\
\hline \multicolumn{5}{|l|}{ Water and sanitation } \\
\hline $\begin{array}{l}\mathrm{HH} \text { with access to safe } \\
\text { water }\end{array}$ & $74.0 \%$ & $64.8-97.7$ & $75.1 \%$ & $67.2-82.6$ \\
\hline $\begin{array}{l}\text { HH with access to any } \\
\text { latrine }\end{array}$ & $34.7 \%$ & $29.1-40.8$ & $55.3 \%$ & $46.4-63.8$ \\
\hline \multicolumn{5}{|l|}{ Household assets } \\
\hline HH owns any radio & $45.9 \%$ & $39.3-52.7$ & $63.6 \%$ & $57.8-69.1$ \\
\hline $\begin{array}{l}\text { HH owns any mobile } \\
\text { phone }\end{array}$ & $53.7 \%$ & $47.0-60.3$ & $60.5 \%$ & $55.2-65.5$ \\
\hline $\begin{array}{l}\text { HH has any means of } \\
\text { transport }\end{array}$ & $51.4 \%$ & $46.2-56.6$ & $60.3 \%$ & $54.9-65.5$ \\
\hline
\end{tabular}

0.2. Sampling of both clusters and households was independently repeated for each round. Surveys were implemented by a local research firm, integrity research and consultancy, using experienced supervisors and interviewers and applying standard measures of quality assurance and supervision. The data collection tool was based on the Malaria Indicator Survey questionnaire with additions to reflect the specific source of nets and the perceptions of the distribution scheme by the population.

Data from the paper-questionnaires were doubleentered and validated using CSPRO software and then transferred for analysis to the Stata software package (version 11). Chi squared test was used for dichotomous or grouped variable comparisons and Student's $t$ test or non-parametric Kruskal-Wallis test for continuous variables, always adjusting for the cluster-design of the survey. In order to account for potential confounders in the analysis of key outcomes, multivariable regression models were used, logistic regression for dichotomous outcomes and linear regression for continuous variables. Models were constructed using backwards elimination and Wald tests for significant parameters.

Primary outcome measures were those of net and ITN ownership as recommended by the Roll-Back Malaria Monitoring and Evaluation Reference Group (RBMMERG) namely the 'proportion of households with any ITN', the 'proportion of households with at least one ITN for every two people in the household' (considered to be enough so that every member could use an ITN) and the 'proportion of the population with access to an ITN within the household' assuming each ITN is used on average by two people [13]. Counterfactual ownership coverage was calculated by only using nets identified as from the campaign or routine distribution, respectively. In the same way, the 2011 post-campaign coverage was estimated from the number of campaign nets received from the campaign reported at the baseline survey.

To evaluate equity of net ownership indicators, a wealth index was created applying principal component analysis (PCA) and using household amenities, livestock, assets, and other characteristics relevant to household socioeconomic status in South Sudan as input variables. The first component was used as wealth index and households grouped into wealth quintiles. Concentration indices were calculated to quantify equity. The concentration index utilizes data from all wealth quintiles and expresses perfect equity as 0 , maximum 'pro-poor' inequity as -1 and maximum 'pro-rich' inequity as +1 . Standard errors and confidence intervals for the concentration indices were calculated using the formula suggested by Kakwani et al. [14].

Community-level ITN coverage was estimated for each survey cluster applying the Lot-Quality-Assurance-Sampling-based methodology (LQAS) suggested by Biedron et al. [15]. In short, each cluster was considered a lot and a pass-fail assessment was made for different ITN coverage targets based on LQAS decision rules based on a 20\%-point margin between target and minimally acceptable result (90/70, 80/60\% etc.).

Data on programme costs were collected and made available by Malaria Consortium's finance department. Costs were categorized as either direct costs, indirect costs or evaluation costs. These were further divided into sub-categories following WHO recommendations and previous costing work by Kolaczinski et al. [16]. Costs were initially reported in GBP, and were converted to USD using an average exchange rate for the period of USD 1.60 per GBP. No cost from the County or national level from the Government of The Republic of South Sudan were available for inclusion so that costing only 
reflects funding by the donors (donor perspective). In addition, only financial and not economic cost estimated was used.

\section{Results}

\section{Coupons and ITNs issued}

Over the 11-month pilot a total of 30,530 coupons were issued to beneficiaries and 28,696 ITNs were distributed, resulting in an overall redemption rate of $94.0 \%$. As shown in Fig. 2, output was quite homogeneous over time with on average 3188 ITNs issued per month. Redemption rate varied between $99.1 \%$ in the second month and $86.9 \%$ in January 2013 when the issuing of coupons ended. For $81.5 \%$ of coupons a reason for issuing was stated: $57.1 \%$ were given because there were not enough ITNs in the household; $31.8 \%$ to replace damaged nets; and, $11.2 \%$ to women who had given birth but had not received an ITN. Overall, 18.5\% of coupons did not have a reason recorded and neither this rate nor the distribution between the recorded reasons varied significantly during the course of the pilot.

\section{Evaluation surveys}

Out of the targeted sample size of 600 households the baseline survey achieved 599 and the endline 597. The corresponding numbers of household members registered in these households were 3567 and 3095, respectively. The setting is a very rural environment where almost all houses have a thatched roof and mud walls, the predominant fuel for cooking is firewood, and the educational level of the mainly male heads of household is relatively low. About three-quarters of the households had access to safe drinking water, mainly from boreholes, and one-third at baseline and half at endline had access to any form of latrine. Both access to safe water and latrines was strongly associated with increased wealth $(\mathrm{p}<0.001)$. Household assets such as radios, mobile phones or means of transport also showed a strong poor-rich gradient $(\mathrm{p}<0.001)$ but also some improvement between the two surveys 12 months apart. The reason for this increase in assets is not clear but did not change the poor-rich gradient from baseline to endline survey. There were also some demographic differences between the samples with a slightly lower average

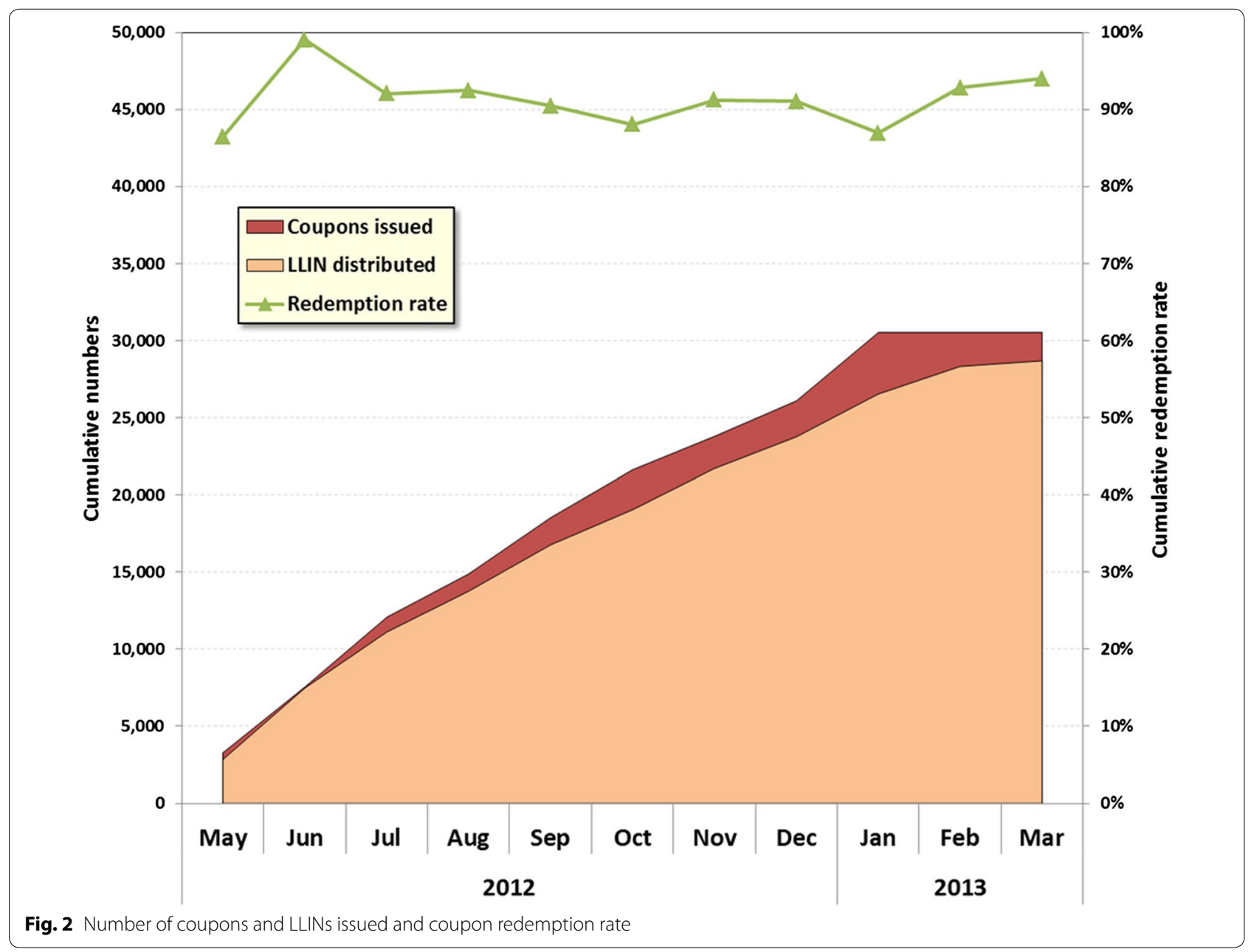


household size at endline $(\mathrm{p}<0.05)$, lower proportion of children under five in the population $(\mathrm{p}<0.05)$ and subsequently a lower person-to-sleeping place ratio $(\mathrm{p}<0.05)$. From the data it cannot be concluded with certainty whether these were based on changes in population composition (e.g., due to migration) or slightly different composition of the sample by chance.

\section{Access to ITNs via the community-based distribution}

At endline $78.3 \%$ of households were aware of the continuous distribution scheme. Community members had heard about it through social mobilizers (77.0\%), net coupon holders (71.3\%), distribution supervisors (28.9\%), clergymen (21.7\%), and/or health workers (18.5\%). Boma chiefs, village health committee members, neighbours, and other officials were reported as sources of information by less than $10 \%$ of households. Most households aware of the scheme also requested an ITN coupon during the 9 months of operation (95.5\%); most of these (95.6\%) received at least one coupon, almost all of those (99.1\%) went to redeem the coupon, and $98.1 \%$ of those also received an ITN. As shown in Table 2, the overall community effectiveness of households receiving any ITN through the scheme was 69.9 , and $88.7 \%$ of those aware of the campaign ended up receiving at least one ITN through the scheme. Stratifying by wealth quintile, there was a certain level of pro-rich inequity $(\mathrm{p}=0.003)$, which had its basis in a lower number of the poorest wealth quintiles hearing about the scheme and then increasing at each step slightly. But as seen in Table 2 from the concentration indices this inequity was moderate and receiving an ITN if aware of the scheme was quite equitable (concentration index 0.03) even though the trend by wealth quintile was still statistically significant.
Only 20 of the 597 households surveyed at endline reported not receiving a coupon despite attempting to acquire an ITN. Of those, thirteen were unable to find a net coupon holder, four households were ineligible based on the established criteria, and four were uncertain why they had not been issued a coupon. Eight households unsuccessfully attempted to obtain nets from distribution points. Four of the eight came when the person responsible for distribution was unavailable, three came during stock outs, and one arrived after the closure of a health facility.

\section{ITN ownership and use-before and after}

At baseline, 773 mosquito nets were registered in surveyed households of which $91.5 \%$ could be identified as LLINs based on brand, three $(0.4 \%)$ were reported as having been dipped with an insecticide within the last 12 months and $8.1 \%$ were untreated nets. At endline there were 1251 nets registered with $99.0 \%$ identified as LLINs and 1.0\% untreated. The changes in ownership and use indicators are shown in Table 3 and demonstrate significant improvements in all areas. As one would expect, the smallest increase of 1.2-fold (from 66.3 to $81.7 \%$ ) was seen in the proportion of households with any ITN, while the population with access to an ITN within the household almost doubled with a 1.8-fold increase (37.9 to $66.2 \%)$. The proportion of households with enough ITNs for all members (at least one ITN for every two people) increased 2.5-fold (from 18.5 to $45.6 \%$ ). This was accompanied by significant reduction in households with some, but clearly not enough ITNs $(<1$ ITN for every three people) from $52.9 \%$ at baseline to $23.2 \%$ at endline. In contrast, the proportion of households with more than enough ITNs remained low (4.5 and 8.8\%, respectively).

Table 2 Effectiveness and equity of community-based distribution scheme components

\begin{tabular}{|c|c|c|c|c|c|c|}
\hline $\begin{array}{l}\text { Background } \\
\text { characteristic }\end{array}$ & $\begin{array}{l}\text { Heard about } \\
\text { coupon }\end{array}$ & $\begin{array}{l}\text { Requested } \\
\text { coupon }\end{array}$ & $\begin{array}{l}\text { Received } \\
\text { coupon }\end{array}$ & $\begin{array}{l}\text { Went to redeem } \\
\text { coupon }\end{array}$ & Received ITN & $\begin{array}{l}\text { Received ITN if heard } \\
\text { about coupon }\end{array}$ \\
\hline \multicolumn{7}{|l|}{ Endline } \\
\hline Estimate (\%) & 78.3 & 75.2 & 71.9 & 71.2 & 69.9 & 88.7 \\
\hline $95 \% \mathrm{Cl}$ & $72.6-83.8$ & $68.3-81.1$ & $64.1-78.5$ & $63.4-77.9$ & $61.6-77.0$ & $82.4-93.0$ \\
\hline \multicolumn{7}{|l|}{ Wealth index } \\
\hline Lowest (\%) & 67.2 & 60.5 & 56.3 & 55.5 & 53.8 & 80.0 \\
\hline Second (\%) & 79.8 & 74.8 & 72.3 & 71.4 & 70.6 & 88.4 \\
\hline Third (\%) & 75.0 & 72.5 & 68.3 & 66.7 & 64.2 & 85.6 \\
\hline Fourth (\%) & 83.2 & 79.8 & 77.3 & 77.3 & 77.3 & 92.9 \\
\hline Highest (\%) & 88.3 & 88.3 & 85.0 & 85.0 & 83.3 & 94.3 \\
\hline $\begin{array}{l}\text { p value Chi squared for } \\
\text { trend }\end{array}$ & 0.003 & $<0.001$ & $<0.001$ & $<0.001$ & $<0.001$ & 0.003 \\
\hline \multicolumn{7}{|l|}{ Concentration index } \\
\hline Estimate & 0.046 & 0.065 & 0.070 & 0.073 & 0.075 & 0.029 \\
\hline $95 \% \mathrm{Cl}$ & $0.023-0.070$ & $0.038-0.091$ & $0.049-0.098$ & $0.044-0.102$ & $0.046-0.105$ & $0.010-0.048$ \\
\hline
\end{tabular}


Table 3 Net and ITN ownership and use before and after the community-based distribution

\begin{tabular}{|c|c|c|c|c|}
\hline \multirow[t]{2}{*}{ Category/indicator } & \multicolumn{2}{|l|}{ Baseline } & \multicolumn{2}{|l|}{ Endline } \\
\hline & Estimate & $95 \% \mathrm{Cl}$ & Estimate & $95 \% \mathrm{Cl}$ \\
\hline \multicolumn{5}{|l|}{ Household ownership } \\
\hline Proportion with any net & $74.3 \%$ & $67.6-80.0$ & $82.4 \%$ & $77.4-86.5$ \\
\hline Proportion with any ITN & $66.3 \%$ & $60.1-71.9$ & $81.7 \%$ & $76.8-85.8$ \\
\hline $\begin{array}{l}\text { Proportion with at least } 1 \\
\text { ITN/2 people }\end{array}$ & $18.5 \%$ & $15.2-22.5$ & $45.6 \%$ & $39.2-52.0$ \\
\hline $\begin{array}{l}\text { Mean number of ITNs if } \\
\text { any owned }\end{array}$ & 1.79 & $1.65-1.92$ & 2.54 & $2.39-2.68$ \\
\hline \multicolumn{5}{|l|}{ Supply with ITN if any owned } \\
\hline$<1$ ITN/3 people & $52.9 \%$ & $47.3-58.4$ & $23.2 \%$ & $17.7-29.6$ \\
\hline 1 ITN/3 people & $19.1 \%$ & $14.8-24.4$ & $21.1 \%$ & $17.4-25.3$ \\
\hline 1 ITN/2 people & $23.4 \%$ & $18.9-28.6$ & $46.9 \%$ & $41.1-52.9$ \\
\hline 1 ITN/person or more & $4.5 \%$ & $2.8-7.3$ & $8.8 \%$ & $6.2-12.4$ \\
\hline \multicolumn{5}{|l|}{ Population (de-facto) access } \\
\hline $\begin{array}{l}\text { Proportion with access to } \\
\text { an ITN }\end{array}$ & $37.9 \%$ & $33.3-42.7$ & $66.2 \%$ & $61.2-70.9$ \\
\hline \multicolumn{5}{|l|}{ Population use of ITNs } \\
\hline Used ITN last night & $22.7 \%$ & $18.0-28.3$ & $53.9 \%$ & $49.6-58.2$ \\
\hline Used ITN if access & $60.1 \%$ & $55.3-64.9$ & $81.5 \%$ & $76.4-86.1$ \\
\hline
\end{tabular}

The proportion of the population using an ITN last night increased from $22.7 \%$ at baseline to $53.9 \%$ at endline. The proportion of household members using an ITN if they had access within the household increased 1.4-fold reaching $81.5 \%$ at endline (Table 3 ). In addition, there was a clear increasing prioritization of ITN use from baseline to endline for children under five and women in reproductive age. Dividing the population into three groups (under-fives, women age 15-49 years, heads of household) and comparing these against the rest of household members, a logistic regression analysis adjusted for wealth and whether a household owned enough ITNs for all members showed an adjusted odds ratio (OR) for ITN use the previous night for children under five of 2.3 at baseline (95\% CI 1.6-3.4) increasing to 5.1 (3.7-7.1) at endline. The increase for women in reproductive age was not quite as strong with adjusted OR 2.2 (1.7-3.0) at baseline and 2.9 (2.-3.6) at endline. Heads of households were also more likely to use an ITN with the adjusted OR increasing from $2.6(2.1-3.3)$ at baseline to $3.7(2.9-4.8)$ at endline. Other factors that had a positive association with ITN use at endline were discussing net use in the household (adjusted OR 6.1, 4.7-13.7) and the expressed intention to use the nets every day (adjusted OR 3.1, 1.27.8). The latter were linked to behavioural change communication (BCC) activities.

\section{Behaviour change communication}

Social mobilization began three months prior to the launch of the pilot, and net coupon holders and social mobilizers delivered ITN messaging throughout the pilot. At baseline, $44.2 \%$ of all households reported receiving information on ITNs or ITN use, with health workers (33\%) and radio (28\%) as the two most common sources of information. Over the course of the pilot, social mobilizers (65\%) and net coupon holders (58\%) became the primary sources of ITN information for all households. Overall, 58.1\% of households had received ITN information by the end of the pilot, a statistically significant increase $(\mathrm{p}<0.05)$. Among households who had actively participated in the community distribution scheme, $79 \%$ of household who obtained at least one net coupon through this channel were exposed to ITN messaging.

Households exposed to ITN messaging were significantly more likely to have discussed net use (98 vs $56 \%$, $\mathrm{p}<0.0001$ ) and expressed their intention to use an ITN regularly (95 vs $48 \%, \mathrm{p}<0.0001$ ). As has been presented above, these two variables were also directly linked to actual use suggesting that the BCC activities in combination with ITN availability contributed to the increased ITN use.

\section{Perceptions of community-based distribution scheme}

Among households who were aware of the distribution scheme, $63.6 \%$ felt that the distance to the net coupon holder was either acceptable or very close. The remaining $36.4 \%$ believed the nearest net coupon holder was too far. Distribution sites were perceived to be slightly less accessible, with only $47.8 \%$ of participants deeming sites as very close or an acceptable distance. Of the five payams, central Lainya payam households generally felt that both net coupon holders and distribution sites were more accessible than the four peripheral payams. Perception of distance was also affected by whether or not a household participated in the community distribution scheme, with $63 \%$ of those who were aware of the campaign but elected not to pursue a coupon feeling that net coupon holders were not close enough, while only $35 \%$ of those who requested and received a coupon felt the distance was too far $(\mathrm{p}=0.04)$.

The communities and households largely felt that the criteria for eligibility were fair, with $88.0 \%$ replying very fair $(63.2 \%)$ or fair $(24.8 \%)$. Similar to perceived distance to a net coupon holder, this was also skewed by participation in the distribution scheme. Only $61 \%$ of households who did not receive a coupon felt the criteria were fair, compared to $91 \%$ among those who did receive a coupon $(\mathrm{p}<0.005)$. 


\section{Equity}

As presented in Table 2, households in the poorer wealth quintiles were less likely to request a new ITN through the community-based distribution. The poorest quintile were as likely as wealthier quintiles to receive an ITN if they received a coupon, but much less likely to know about community distribution, or request a coupon if they did know about the scheme than the wealthier quintiles. A closer look at equity of ITN ownership is presented in Fig. 3, showing that the gradient between wealth quintiles for coverage with at least one ITN remained nearly unchanged between baseline and endline survey, i.e., the curve only shifted upward. However, equity for enough ITNs (at least one ITN for two people) showed a significantly more pro-rich distribution. This is also reflected in the concentration indices which were identical, 0.041 and 0.040 , for households owning any ITN at baseline and endline, respectively. The concentration indices increased from 0.002 to 0.144 ( $\mathrm{p}<0.05$ ) for households with enough ITNs.

\section{Community coverage}

Results from the LQAS approach to assess communitylevel coverage are presented in Fig. 4, showing that community-based distribution substantially raised community coverage for household ownership of any ITN, and households with enough ITNs. At baseline, nearly all (>95\%) communities achieved a level of $50 \%$ of households with at least one ITN, but only $40 \%$ of communities attained the national target of $80 \%$ household ITN ownership. Almost none (3\%) of the communities surveyed at baseline met the target of having $50 \%$ of households with enough ITNs. At endline, about $85 \%$ of communities were found to have over $80 \%$ household ownership of at least one ITN, and more than $60 \%$ of communities had half of households owning enough ITNs. As Fig. 4 shows, there were noticeable gains at each five percentage point marker up to $80 \%$ for households with enough ITNs.

\section{Other sources of nets and ownership trends over time}

At endline, $69.9 \%$ of households had received an ITN through the community-based distribution scheme, and $95.9 \%$ of those still owned these nets. Other distribution channels that households received ITNs from included routine ante-natal care services (ANC) (15.4\%), the previous mass distribution campaign (12.4\%), and the retail market (10.1\%). Utilization of the community-based distribution scheme varied based on whether a household did or did not receive ITNs through the previous mass campaign, with $70 \%$ of households that did not receive any ITNs through the mass campaign receiving ITNs through community-based distribution, compared

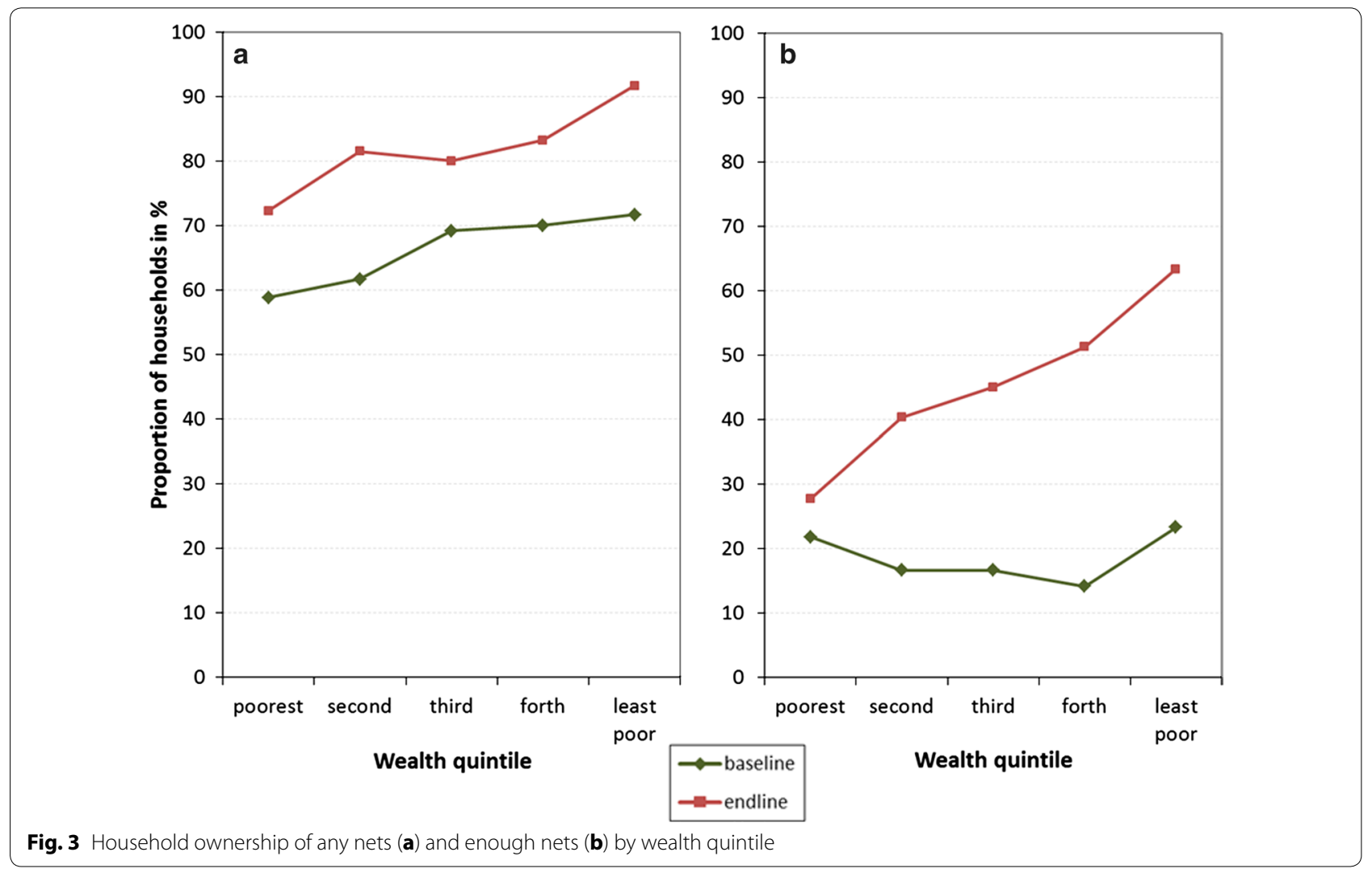



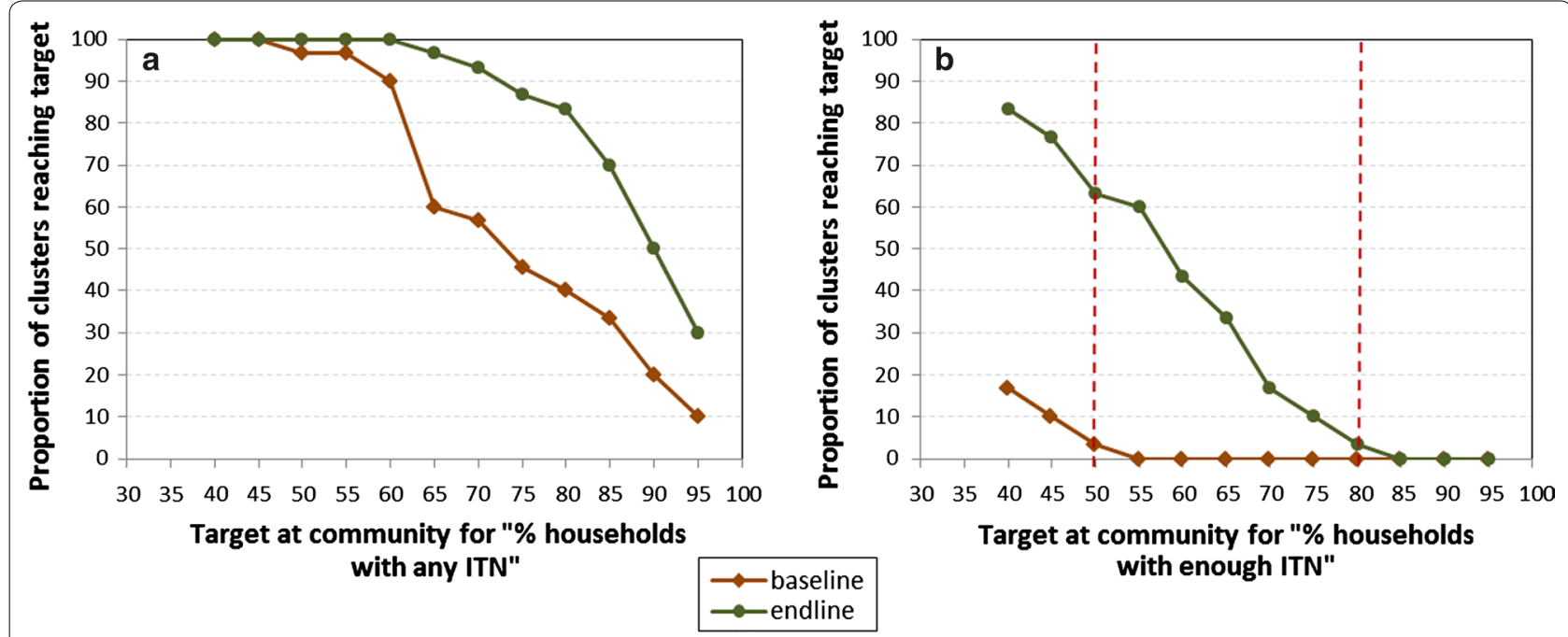

Fig. 4 Community level coverage estimates for households with any ITN (a) and enough ITN (b)

to $50 \%$ of households that did receive nets through the prior mass campaign. Households with women attending ANC at a health facility were less likely (56\%) to receive an ITN through community distribution than households not attending ANC. Following the mass campaign, the community-based distribution served as the only source of new ITNs for more than half of households (53.4\%). Nets from multiple channels were obtained by only $15 \%$ of households and the most common combination was community scheme plus ANC (8.4\%) followed by community scheme plus nets bought from private sector (5.7\%). Only $6 \%$ received new ITNs only from ANC.

The trend in ITN ownership coverage from the campaign in 2011 up to the endline survey in 2013 is shown in Fig. 5, giving the overall result as well as the counterfactual of what coverage would be observed without the contributions of the $C D$ pilot, and only considering ITNs remaining from the campaign and private sector. The 2011 post campaign results are estimated from the baseline survey based on campaign nets received reported by the households. Results show that the proportion of households with any ITN increased moderately from $64 \%$ at the time of the mass campaign to $66 \%$ at the start of the CD-pilot and then increased sharply to $81 \%$ at the endline survey. In contrast, the proportion of households with at least one ITN for every two people slightly decreased after the campaign from 21 to 19\% but then sharply increased to $46 \%$ at endline. Excluding

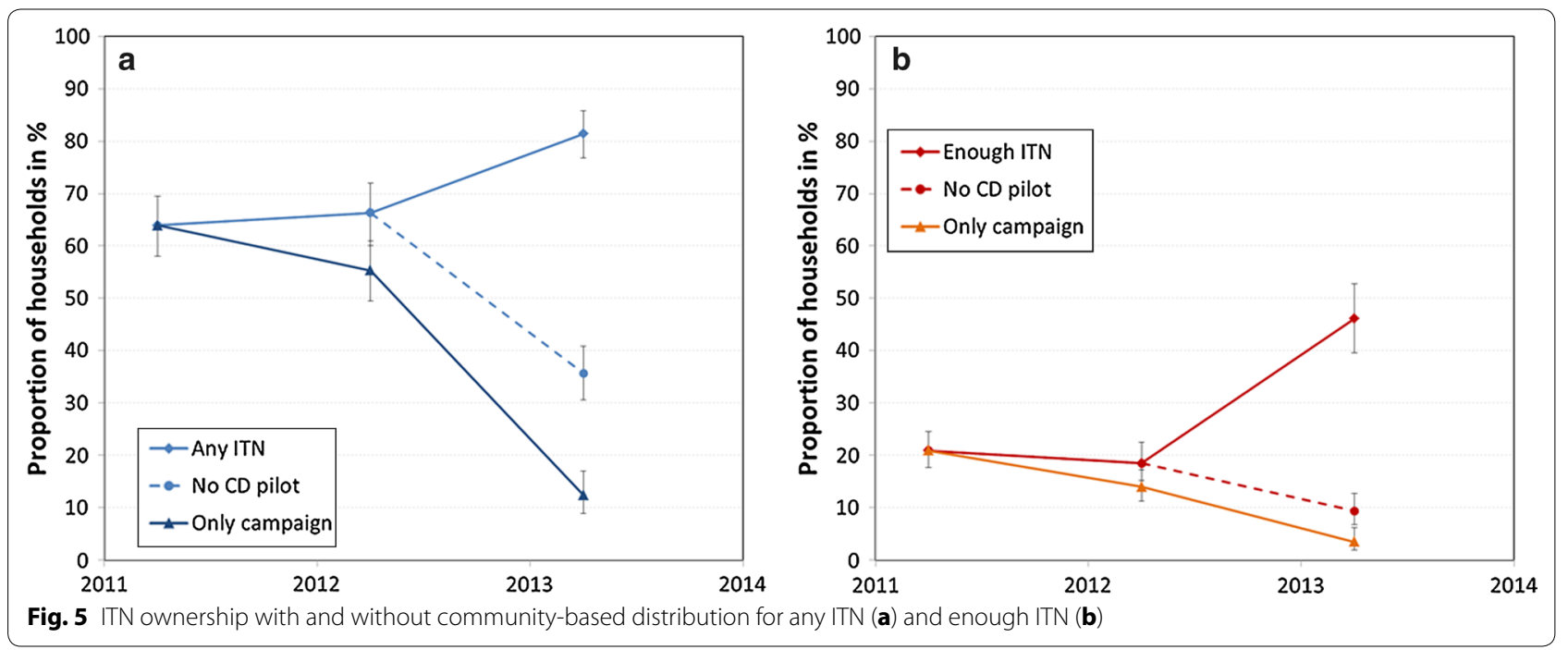


community-based distribution would have resulted in coverage of $36 \%$ with any ITN and only $9 \%$ with at least one ITN for every two people in 2013. There also was a clear difference between only campaign nets and coverage without the community-based distributions showing that nets from ANC, private sector and 'other sources', i.e., friends, family and civil society, contributed to postcampaign coverage but not enough to prevent decline of coverage rates.

\section{Cost}

The financial cost of the community-based distribution scheme in Lainya County was composed of direct, indirect and household survey costs. Total cost for all three categories was USD667,299 and USD551,318 after removing the cost of surveys resulting in cost per ITN distributed of USD19.21 and USD16.52 when the procurement of the ITN was excluded. Indirect cost for staff employed by the implementer to support the scheme and their travel and management cost was high and made up $49 \%$ of the total. Direct cost alone was USD9.79 per net delivered and USD7.02 without ITN procurement. Of the direct cost the largest proportion (58.7\%) went to operations with transport (vehicle hire and fuel) being the largest component with $27.6 \%$ of direct cost followed by training, mobilization and $\mathrm{BCC}(12.1 \%)$, net storage $(9.3 \%)$ and supervision (7.8\%). Incentives for the storekeepers, including airtime for communication, comprised $9.4 \%$ of direct cost (details of cost categories are shown in Additional file 1).

Plotting cumulative cost per net delivered against the months of programme operation showed a very high initial cost based on the high spending during roll-out of USD31.30 (without ITN procurement) in the first month which then rapidly declined in the next 3 months to USD10.59 and then slowly further declined to reach a steady state around USD6.55 after 9 months of implementation.

\section{Discussion}

Community-based distribution of ITNs in Lainya County over a period of 9 months in conjunction with additional ITN distribution through ante-natal care services (ANC) and purchases from the retail market by some households significantly increased population access to ITNs from $38 \%$ one year after a mass distribution campaign to $66 \%$ at the end of the pilot. Household ownership of any ITN at endline was $82 \%$ and $46 \%$ of households owned enough ITNs for all household members (one ITN for every two people). In spite of the increases the target for universal coverage of $80 \%$ population ITN access was not quite achieved. This was in part due to the lower than expected coverage remaining from the mass campaign of 2010.
Awareness and acceptability of the scheme were high. In addition, the endline survey results showed a high level of community coverage where $85 \%$ of communities (clusters) had at least $80 \%$ of households with any ITN and $60 \%$ had at least half of households with enough ITNs for all household members. Although the exact minimal and optimal coverage levels for the ITN mass effect are not precisely defined, available evidence suggests $[17,18]$ that the observed community coverage level is sufficient for a significant impact on transmission reduction and protection of people without access to an ITN, assuming there is no pyrethroid resistance among the dominant vectors.

A number of descriptions of continuous, communitybased net or ITN distributions can be found in the literature to which the Lainya pilot can be compared. However, most of these were undertaken before universal coverage became the objective of malaria prevention with ITNs. These distributions were either part of implementation research, smaller scale projects or social marketing approaches. Community health workers or community leaders have been used to selling subsidized nets in Afghan refugee camps in Pakistan [19], in several communities in Latin America [20], and in social marketing projects in Kilombero, Tanzania [2] and Sofala and Manica Provinces in Mozambique [21]. Communitybased organizations, such as committees or cooperatives, have been used for the same purpose in four areas in Kenya [22], and Mexico and Colombia [23]. All these examples show that a demand-driven ITN distribution scheme is feasible and acceptable to the communities, but using these approaches alone and without building on a previous mass campaign, ITN ownership only increases gradually [3]. Only one paper describes the outcome of a community-based ITN distribution that had the objective to reach universal ITN coverage. In a health district in Western Niger community health committees were supported to sell subsidized ITNs (USD1.20 to the consumer) to all interested households resulting in ITN ownership of at least one ITN of $83 \%$ compared to $61 \%$ in a control area [24]. Given that ITNs were sold and not given free as in Lainya County, this success is very similar to what was reported in this study even though not all ownership indicators are reported and no baseline was undertaken.

The present pilot provides proof-of-principle that a community-based ITN distribution scheme can reach sufficient number of households to sustain and even increase ITN coverage thereby confirming the study hypothesis. This is demonstrated by several findings. First, with $96 \%$ of households who were aware of the scheme requesting additional ITNs at least once, households showed that they not only were aware of the need for more nets, but also were ready to take the initiative 
to get them. Second, after the addition of distribution points in remote areas, the density and functionality of access points was sufficiently high for people to obtain ITNs. Less than $1 \%$ of households received a coupon but did not go to a redemption point, and only another $1 \%$ did not get a net because of stock-out or other reasons, resulting in a $94 \%$ redemption rate. This suggests that acceptability in the community was high enough for this system to continue at a similar level provided demand was met with supply. The lower awareness of the scheme among households in the lowest wealth quintile, however, is more problematic. This resulted in a pro-rich gradient especially for the indicator for 'enough' ITNs for all household members (concentration index 0.14). Although the inequity was moderate compared to other observations on ITNs or immunization coverage from African countries [25], it was significant enough to raise concern and in contrast to the high level of equity generally found following mass campaigns [3, 7]. Future interventions should establish additional social mobilization targeted especially at this group to ensure they are empowered to request sufficient numbers of ITNs to protect their families keeping in mind, however, that this is likely to further increase cost. Third, there were no attempts observed to trick the system or fraudulently obtain nets and $88 \%$ of households thought the system to be fair or very fair.

Lainya County is a challenging, rural operating environment with limited resources and a poorly functioning health system. Accordingly, significant efforts were needed by the implementation partner to initially set the scheme up and provide the necessary supervision and support to run it, resulting in an overall direct and indirect financial cost from the donor perspective of USD16.50 per net delivered without ITN procurement. At a steady state after 9 months, the cost was around USD6.50 per net delivered which is still significantly higher than the USD1.00-1.50 found for mass campaigns $[10,16,26]$. However, these cost estimates may not be comparable for a number of reasons. First, cost estimation methodologies have been found to lack standardization [27], which is especially true for the levels of contributions from other sources than the donor, such as staff time of government employees or use of government vehicles. In the case of the pilot described here these contributions were minimal, thereby increasing the contribution by the donor. Second, although higher than the cost of an average mass campaign, cost of the pilot were not far from those found for a variety of noncampaign distribution approaches such as health facilitybased schemes and social marketing where the annual economic costs for an assumed 3 year life span of the nets ranged from USD3.50 to 7.75 [28]. In addition, it can be expected that in the difficult environment of South Sudan cost for a mass campaign would also exceed the figures quoted above. Unfortunately, such cost estimate is not available. Third, the primary objective of the pilot was to provide evidence that a community-based approach can work in a difficult environment. Supervision was intense: cars were hired from the private sector, and incentives were given to storekeepers to ensure they were motivated to participate in an activity that was not part of their routine tasks at the time. Finding the optimal point between increased efficiency through operational cost savings and the danger of decreased cost-effectiveness through diminished impact is always a challenge and in general this process occurs during routine implementation and not at the pilot stage. While it is likely that in such routine implementation donor cost per net delivered would further decrease, it is impossible to say by how much. Finally, one must keep in mind that the cost presented here is only the financial cost of delivery from the donor perspective and do not take into account programme effects and benefits over the lifetime of the ITN. A recent analysis of system efficiencies of ITN distributions in Africa suggests that up to $21 \%$ of ITNs may be over-allocated by the current practice of mainly repeat campaigns with some distributions through routine health services [11]. It is, therefore, possible that a continuous distribution through a community scheme that builds on a pushpull mechanism may reduce such inefficiencies. However, cost-benefit analysis in a routine implementation setting would be needed to provide such evidence.

There were two key limitations of this study. As mentioned previously, during the implementation of this pilot special efforts were put into regular supervision and monitoring. This prevents generalizing the positive results to more routine settings or implementation within the general health system where high motivation of staff and additional resources may not be available. Secondly, the relatively short length of the pilot ( 9 months) precludes drawing any conclusions about the longer-term ability of the scheme to maintain ITN ownership and access.

\section{Conclusion}

The Lainya County pilot provides evidence that a demand-driven community-based distribution can not only sustain ITN coverage levels obtained through a mass campaign but also increase population ITN access across communities and improve the number of households with enough ITNs for all household members. Longer periods of implementation should be evaluated to determine whether community-based distribution can successfully maintain ITN coverage beyond the short term, and reach all wealth quintiles equitably. 


\section{Additional file}

Additional file 1. Detailed cost of pilot implementation.

\section{Authors' contributions}

AK, LWS, TM, AS, KL, MZG, RA, SM, and GG were involved in study design and implementation. AK, LW and TM analysed the data, LW, AK, SB, HK, MF, LG, and $\mathrm{ML}$ drafted and edited the manuscript for publication. All authors read and approved the final manuscript.

\section{Author details}

${ }^{1}$ Malaria Consortium, London, UK. ${ }^{2}$ Tropical Health LLP, Montagut, Spain. ${ }^{3}$ Malaria Consortium, Juba, South Sudan. ${ }^{4}$ Johns Hopkins University Center for Communication Programs, Baltimore, USA. ${ }^{5}$ President's Malaria Initiative, U.S. Agency for International Development, Washington D.C., USA. ${ }^{6}$ President's Malaria Initiative, U.S. Agency for International Development, Dar es Salaam, Tanzania.

\section{Acknowledgements}

The authors would like to thank all of the study participants, Lainya County Health Department, staff, health facility staff, and community health workers for their significant contribution to this research and manuscript. In addition the following persons provided much appreciated support: Harriet Pasquale and Richard Lako from the Ministry of Health of South Sudan.

\section{Competing interests}

The authors declare that they have no competing interests.

\section{Availability of data and materials}

Data analysed and used in this study and reported in this manuscript are available from the corresponding author per reasonable requests.

\section{Consent for publication}

Not applicable.

\section{Ethics approval and consent}

Ethical approval was received from the Division of Monitoring, Research, Evaluation, and Research of the Ministry of Health of the Republic of South Sudan on 19 March, 2012. All questionnaire respondents offered verbal consent prior to interview, and were made aware of the purpose of this study and their right to refuse.

\section{Funding}

This work was funded through a DFID Programme Partnership Arrangement with co-funding made possible by the generous support of the American people through the US Agency for International Development (USAID) and the President's Malaria Initiative (PMI) under the terms of USAID/JHU Cooperative Agreement No. GHS-A-00-09-00014-00 (NetWorks Project) and AID-OAAA-14-00057 (VectorWorks Project). The contents are the responsibility of the authors and do not necessarily reflect the views of USAID/DFID or the United States or United Kingdom Governments.

\section{Publisher's Note}

Springer Nature remains neutral with regard to jurisdictional claims in published maps and institutional affiliations.

Received: 1 August 2017 Accepted: 6 September 2017 Published online: 11 September 2017

\section{References}

1. Spencer S, Grant AD, Piola P, Tukpo K, Okia M, Garcia M, et al. Malaria in camps for internally-displaced persons in Uganda: evaluation of an insecticide-treated bednet distribution programme. Trans R Soc Trop Med Hyg. 2004;98:719-27.
2. Schellenberg JRMA, Abdulla S, Minja H, Nathan R, Mukasa O, Marchant T, et al. KINET: a social marketing programme of treated nets and net treatment for malaria control in Tanzania, with evaluation of child health and long-term survival. Trans R Soc Trop Med Hyg. 1999;93:225-31.

3. Kilian A, Wijayanandana N, Ssekitoleeko J. Review of delivery strategies for insecticide treated mosquito nets - are we ready for the next phase of malaria control efforts? TropIKAnet J. 2010;1:1.

4. Grabowsky M, Farrell N, Hawley W, Chimumbwa J, Hoyer S, Wolkon A, et al. Integrating insecticide-treated bednets into a measles vaccination campaign achieves high, rapid and equitable coverage with direct and voucher-based methods. Trop Med Int Health. 2005;10:1151-60.

5. Thwing J, Hochberg N, Eng JV, Issifi S, James Eliades M, Minkoulou E, et al. Insecticide-treated net ownership and usage in Niger after a nationwide integrated campaign. Trop Med Int Health. 2008;13:827-34.

6. WHO. Insecticide treated mosquito nets: a position statement. Global malaria programme. Geneva: World Health Organization; 2007.

7. Zegers de Beyl C, Koenker H, Acosta A, Onyefunafoa EO, Adegbe E, McCartney-Melstad A, et al. Multi-country comparison of delivery strategies for mass campaigns to achieve universal coverage with insecticidetreated nets: what works best? Malar J. 2016;15:1151.

8. Bhatt S, Weiss DJ, Cameron E, Bisanzio D, Mappin B, Dalrymple U, et al. The effect of malaria control on Plasmodium falciparum in Africa between 2000 and 2015. Nature. 2015;526:207-11.

9. Koenker HM, Yukich JO, Mkindi A, Mandike R, Brown N, Kilian A, et al. Analysing and recommending options for maintaining universal coverage with long-lasting insecticidal nets: the case of Tanzania in 2011. Malar J. 2013;12:150.

10. Yukich J, Bennett A, Keating J, Yukich RK, Lynch M, Eisele TP, et al. Planning long lasting insecticide treated net campaigns: should households' existing nets be taken into account? Parasit Vectors. 2013;6:174.

11. Bhatt S, Weiss DJ, Mappin B, Dalrymple U, Cameron E, Bisanzio D, et al. Coverage and system efficiencies of insecticide-treated nets in Africa from 2000 to 2017. eLife. 2015:4:e09672.

12. RBM Vector Control Working Group. Consensus statement on continuous distribution systems for insecticide treated nets, June 2011. http://www. rollbackmalaria.org/files/files/partnership/wg/wg_itn/docs/vcwg6report1.pdf. Accessed 01 Aug 2017.

13. RBM-MERG. Household survey indicators for malaria control. RBM 2013. http://www.rollbackmalaria.org/files/files/toolbox/docs/rbmtoolbox/ tool_HouseholdSurveyIndicatorsForMalariaControl.pdf. Accessed 01 Aug 2017 .

14. Kakwani N, Wagstaff A, van Doorslaer E. Socioeconomic inequalities in health: measurement, computation, and statistical inference. J Econom. 1997;77:87-103.

15. Biedron C, Pagano M, Hedt BL, Kilian A, Ratcliffe A, Mabunda S, et al. An assessment of lot quality assurance sampling to evaluate malaria outcome indicators: extending malaria indicator surveys. Int J Epidemiol. 2010:39:72-9.

16. Kolaczinski JH, Kolaczinski K, Kyabayinze D, Strachan D, Temperley M, Wijayanandana $\mathrm{N}$, et al. Costs and effects of two public sector delivery channels for long-lasting insecticidal nets in Uganda. Malar J. 2010;9:102.

17. Hawley WA, Phillips-Howard PA, ter Kuile FO, Terlouw DJ, Vulule JM, Ombok $M$, et al. Community-wide effects of permethrin-treated bed nets on child mortality and malaria morbidity in western Kenya. Am J Trop Med Hyg. 2003;68:121-7.

18. Killeen GF, Tami A, Kihonda J, Okumu FO, Kotas ME, Grundmann H, et al. Cost-sharing strategies combining targeted public subsidies with privatesector delivery achieve high bednet coverage and reduced malaria transmission in Kilombero Valley, southern Tanzania. BMC Infect Dis. 2007:7:121.

19. Kolaczinski JH, Muhammad N, Khan QS, Jan Z, Rehman N, Leslie TJ, et al. Subsidized sales of insecticide-treated nets in Afghan refugee camps demonstrate the feasibility of a transition from humanitarian aid towards sustainability. Malar J. 2004;3:15.

20. Kroeger A, Meyer R, Mancheno M, Gonzalez M, Pesse K. Operational aspects of bednet impregnation for community-based malaria control in Nicaragua, Ecuador, Peru and Colombia. Trop Med Int Health. 2003:2:589-602.

21. Brentlinger PE, Correia MAC, Chinhacata FS, Gimbel-Sherr KH, Stubbs B, Mercer MA. Lessons learned from bednet distribution in Central Mozambique. Health Policy Plan. 2007;22:103-10. 
22. Wacira DG, Hill J, McCall PJ, Kroeger A. Delivery of insecticide-treated net services through employer and community-based approaches in Kenya. Trop Med Int Health. 2007;12:140-9.

23. Kroeger A, Aviñna A, Ordoñnez-Gonzalez J, Escandon C. Community cooperatives and insecticide-treated materials for malaria control: a new experience in Latin America. Malar J. 2002;1:15.

24. Nonaka D, Maazou A, Yamagata S, Oumarou I, Uchida T, Yacouba HJ, et al. Distribution of subsidized insecticide-treated bed nets through a community health committee in Boboye Health District, Niger. Trop Med Health. 2012;40:125-31.

25. Webster J, Lines J, Bruce J, Armstrong Schellenberg JRM, Hanson K. Which delivery systems reach the poor? A review of equity of coverage of ever-treated nets, never-treated nets, and immunisation to reduce child mortality in Africa. Lancet Infect Dis. 2005;5:709-17.
26. Yukich JO, Zerom M, Ghebremeskel T, Tediosi F, Lengeler C. Costs and cost-effectiveness of vector control in Eritrea using insecticide-treated bed nets. Malar J. 2009:8:51.

27. Kolaczinski J, Hanson K. Costing the distribution of insecticide-treated nets: a review of cost and cost-effectiveness studies to provide guidance on standardization of costing methodology. Malar J. 2006;5:37.

28. Yukich J, Lengeler C, Tediosi F, Brown N, Mulligan JM, Chavasse D, et al. Costs and consequences of large-scale vector control for malaria. Malar J. 2008;7:258.

\section{Submit your next manuscript to BioMed Central and we will help you at every step:}

- We accept pre-submission inquiries

- Our selector tool helps you to find the most relevant journal

- We provide round the clock customer support

- Convenient online submission

- Thorough peer review

- Inclusion in PubMed and all major indexing services

- Maximum visibility for your research

Submit your manuscript at www.biomedcentral com/submit 